\title{
Smarter than an iPhone: the emergence of the modern electron microscope
}

\author{
Ondrej L. Krivanek ${ }^{1,2}$ \\ ${ }^{1 .}$ Nion R\&D, 11511 NE 118th St., Kirkland, WA 98034, USA \\ 2. Department of Physics, Arizona State U., Tempe, AZ 85287, USA
}

MSA - the Microscopy Society of America - was founded in 1942 as EMSA, with the extra "E" standing for "Electron". The first delivery of RCA's transmission electron microscope (TEM, to American Cyanamid) took place in December 1940, and the society's mission was to advance electron microscopy and especially specimen preparation [1]. The resolution of the first TEMs was about $10 \mathrm{~nm}$ - significantly better than the $200 \mathrm{~nm}$ resolution possible at that time with light microscopes, but a far cry from the $0.1 \mathrm{~nm}(1 \AA)$ resolution needed to resolve individual atoms in a wide range of materials.

Improvements over the next few decades proceeded in many stages: correction of first-order astigmatism, decreased aberrations of the objective lens, increased accelerating voltage (for improved penetration of thick samples and higher spatial resolution), brighter electron guns, improved stability of power supplies and sample stages, and improved vacuum (to minimize contamination). By the time of EMSA's $50^{\text {th }}$ anniversary in 1992, electron microscopes had progressed to lattice resolution (information limit) better than $1 \AA$, and point-to-point (directly interpretable) resolution better than $2 \AA$.

Several new types of electron microscopes were introduced and became popular during this period, such as the scanning electron microscope (SEM), the environmental SEM (ESEM) and the scanning transmission electron microscope (STEM). Crewe's brilliant idea - STEM with a cold field-emission gun - had progressed to a practical instrument built in Crewe's laboratory, and in 1970 it led to the first clear images of individual heavy atoms produced by an electron microscope, at about $2.5 \AA$ resolution [2]. Many analytical techniques were developed, with electron beams smaller than $2 \AA$ produced by STEMs and a wide variety of detectors developed for electron energy loss spectroscopy (EELS), energydispersive X-ray spectroscopy (EDXS), Auger spectroscopy, secondary and back-scattered electrons, cathodoluminescense, and several others. Tomography and ptychography were also introduced.

In parallel, new microscopy techniques such as scanning tunneling microscopy (STM) and atomic force microscopy (AFM) were introduced [3] and quickly progressed to atomic resolution. The resolution of optical microscopes also improved, and fluorescence microscopy and other techniques especially well suited to biological imaging were developed. Recognizing these trends, EMSA became the present-day Microscopy Society of America in 1993, but emphasis on electron microscopy remains to this day.

The fundamental TEM (and STEM) resolution limit, given by the spherical aberration of the objective lens and the wavelength of the electron, had been reached by the early 1970s, and further improvements became incremental. The limit was swept away in the 1990s by the introduction of successful aberration correctors $[4,5]$, with the first commercial corrector delivered in 2000. Aberration correction eventually lowered the resolution of electron microscopes about $3 \mathrm{x}$, to better than $0.5 \AA$, and achieved many remarkable breakthroughs, such as atomic resolution at accelerating voltages of $60 \mathrm{kV}$ and less, thus avoiding knock-on radiation damage of elements as light as boron, and more than 10-fold increase in the electron current available in atom-sized electron probes. Other key innovations included fast and large silicon-drift detectors (SDDs) for EDXS, and monochromators allowing $<10 \mathrm{meV}$ EELS resolution. 
The design of electron microscopes has become much more complicated during this time. Instead of the 3 lenses and no stigmators of the RCA model B electron microscope, modern STEMs have many round lenses (the monochomated Nion UltraSTEM has ten), one or more magnetic prisms, and up to several hundred optical elements such as dipoles, quadrupoles, and higher-order multipoles. This complexity is managed by ultra-stable electronics run by software that achieves "pure" effects by linking several optical elements together, analyzes and automatically nulls aberrations up to $5^{\text {th }}$ order, and also checks for defects such as accidentally disconnected electric cables. It has resulted in spectacular advances in our ability to analyze matter, as illustrated by the examples in Figures 1 and 2.

Similar hidden complexity powers much of the modern world: cloud computing, Amazon's Alexa and Apple's Siri, Google's self-driving cars. As one example out of many, Apple's user-friendly iPhones use some 10 separate microprocessors, and their system diagrams resemble a 3-dimensional megapolis.

These wider-world developments are reflected in a push for microscopes to handle all their alignments and calibration checks automatically in the background, and to interact with users in a way that is centred on the scientific problem to solve rather than the microscope's functions. Artificial intelligence is playing an increasing role, and open-source software such as Nion's Swift [6] promises to allow many laboratories to contribute to the overall progress. When the user is able to ask the microscope questions such as "Microscope, what's the composition and chemical bonding in this area?", and the instrument's software responds by selecting and using the optimum technique and acquisition parameters as well as data processing and display to produce results similar to those shown below, we will have fully combined the remarkable power of modern electron optics with the equally remarkable power of modern computing. The microscope will then become a scientific tool whose power to investigate matter will leave more general-purpose "smart assistants" far behind.

[1] http://www.microscopy.org/about/history.cfm\#overview

[2] AV Crewe, J Wall and J Langmore, Science 168 (1970) 1338.

[3] G Binnig et al., Phys Rev Letts 50 (1983) 120; Phys Rev Letts 56 (1986) 930.

[4] M Haider et al. Optik 106 (Suppl. 7) (1997) S7; Nature 392 (1998) 768.

[5] OL Krivanek et al., Inst. Phys. Conf. Ser. 153 (1997) 35; Ultramicroscopy 78 (1999) 1.

[6] http://www.nion.com/swift/

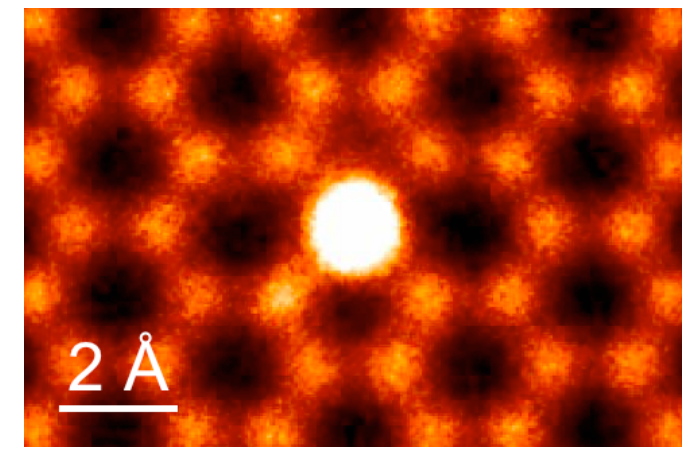

Figure 1. Medium-angle annular dark field image (MAADF) of a single $\mathrm{Si}$ atom replacing two $\mathrm{C}$ atoms in monolayer graphene. Nion UltraSTEM100, $60 \mathrm{kV}$. Courtesy Wu Zhou, ORNL and UCAS.

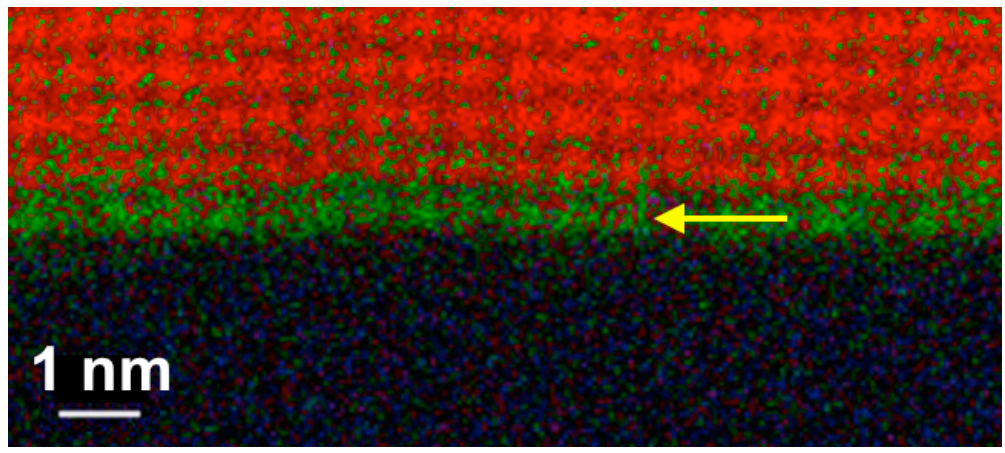

Figure 2. Atomic-resolution bonding map showing the Eu side of a sharp $\mathrm{EuTiO}_{3} / \mathrm{DyScO}_{3}$ interface, obtained by analyzing the chemical shift of Eu $\mathrm{M}_{4,5}$ EELS white lines. $\mathrm{Eu} 2^{+}=$red, Eu $3^{+}=$green (interface monolayer is arrowed). Courtesy Lena Kourkoutis and David Muller, Cornell U. 